Original Article

\title{
Heterogeneity of diabetes - An Indian perspective
}

\author{
Rashmi B. Prasad a, *, Emma Ahlqvist a , Leif Groop a, b \\ a Department of Clinical Sciences, Clinical Research Centre, Lund University, Malmö, Sweden \\ ${ }^{\mathrm{b}}$ Finnish Institute of Molecular Medicine (FIMM), Helsinki University, Helsinki, Finland
}

Type 2 diabetes is a lifelong debilitating disease that results from the inability to maintain glucose homeostasis in the body. Very often, this can lead to complications resulting in abnormal functioning of kidneys (nephropathy), eyes (retinopathy), nerve damage (neuropathy), heart (cardiovascular disorders) and other organs [1]. Current treatment strategies have only been partially successful in disease management and less successful in preventing complications. This could be partially attributed to limiting the diagnosis based on one measured metabolite i.e. glucose, while the manifestation and progression of diabetes have been largely heterogeneous.

Conventionally, diabetes has been classified as insulin dependent diabetes mellitus (IDDM) now known as type 1 diabetes and non-insulin dependent diabetes mellitus (NIDDM), now referred to as type 2 diabetes. Type 1 diabetes is typically diagnosed at a young age and is now known to be GAD positive. Other types such as the glutamate decarboxylase antibodies positive (GAD + ve) latent autoimmune diabetes of adults (LADA) and monogenic forms including neonatal diabetes, maturity onset diabetes of the young (MODY) and Ketosis-prone diabetes (KPD, a. k.a. Flatbush diabetes) have been described. The remaining are classified as type 2 diabetes, which comprises $>90 \%$ of diabetes cases [1].

Existing treatment guidelines are limited by the fact that they respond to poor metabolic control which is remembered by target tissues for a long time ("metabolic memory"), but do not have means to predict which patients will need intensified treatment [2]. This substantiates a need for a more refined system of classification to identify individuals at the time of diagnosis who are at greatest risk for complications. This will facilitate more individualized therapeutic strategies.

To address this goal, we performed data driven cluster analysis (k-means and hierarchical clustering) in patients newly diagnosed with diabetes from the Swedish All New Diabetes in Scania cohort (ANDIS) based on six commonly measured variables [3]. These include GAD, age at diagnosis, BMI, HbA1c, and homoeostatic model assessment 2 estimates of $\beta$-cell function (HOMA2-B) and insulin resistance (HOMA2-IR). Five clusters were identified and replicated in other studies including the Scania Diabetes Registry

\footnotetext{
* Corresponding author.

E-mail address: rashmi.prasad@med.lu.se (R.B. Prasad).
}

(SDR), All New Diabetics in Uppsala (ANDIU), and Diabetes Registry Vaasa (DIREVA) [3].

These five clusters include (Fig. 1):

1. SAID = Severe Autoimmune Diabetes

GAD antibodies, low insulin secretion, poor metabolic control

2. SIDD $=$ Severe Insulin Deficient Diabetes

Low insulin secretion, poor metabolic control, increased risk of retinopathy

3. SIRD $=$ Severe Insulin Resistant Diabetes Insulin resistance, obesity, late onset, increased risk of nephropathy

4. MOD = Mild Obesity-Related Diabetes

Obesity, early onset

5. MARD $=$ Mild Age-Related Diabetes

Late onset, low risk of complications

Based on this classification, cluster 3 individuals who were most insulin resistant had the highest risk of diabetic kidney disease compared to those in clusters 4 and 5 whereas those in cluster 2 had the highest risk for retinopathy, while all individuals had been prescribed the same treatment. Taking this new information into consideration, it is clear that patients would benefit from different treatment strategies.

In terms of genetics, clear evidence was presented to support the clustering. Known T2D loci associated differently between different clusters including the TCF7L2 SNP, which associated with clusters SIDD, MOD, and MARD, but not SIRD. Another example was that of the T1D associated SNP rs2854275 in the HLA locus, which here is as expected strongly associated with SAID, the autoimmune cluster but not with SIDD, the insulin deficient cluster [3].

It is important to consider that these studies have been performed on the European, specifically, Scandinavian populations. Studies have indicated that the South East Asian population is genetically diverse from the European one. Further, recent genomic studies have indicated that most South Asians are descended from two major ancestral components; the Ancestral South Indian (ASI) which is restricted to south Asia, and the Ancestral North Indian (ANI), which is more closely related to those in Central Asia, West Asia and Europe [4]. 

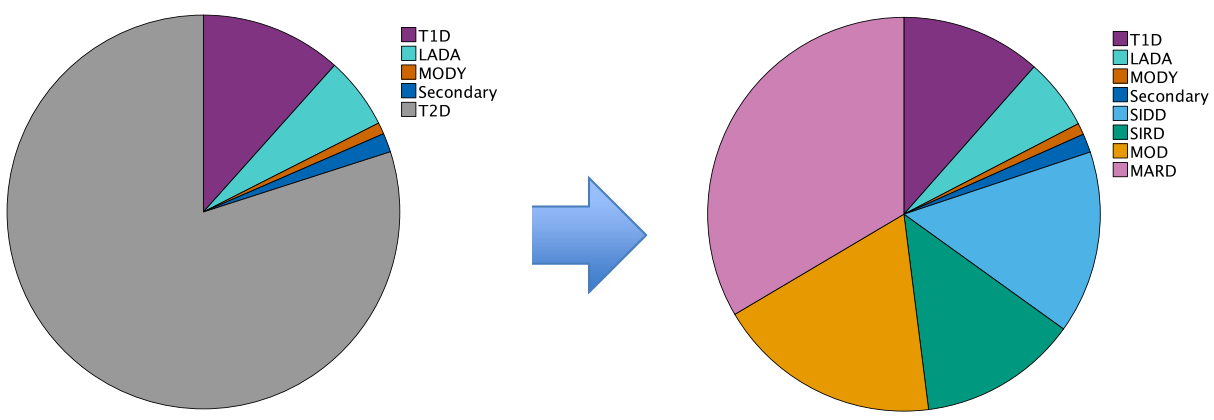

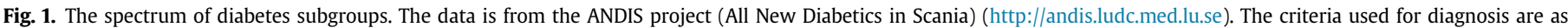

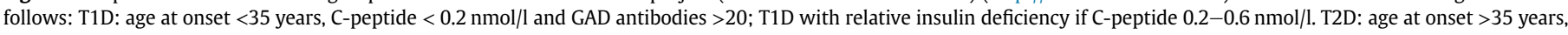

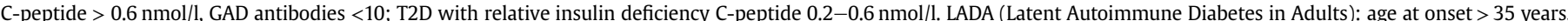

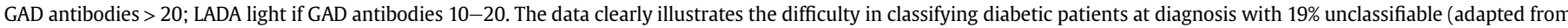

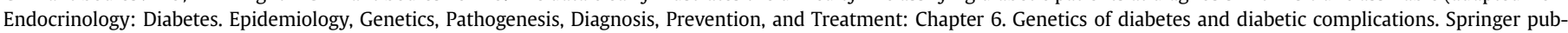
lications 2018).

The Indian population comprises more than one-seventh of the world's population with a great amount of cultural, linguistic and genetic diversity [5]. India is expected to have one of the largest absolute increases in diabetes prevalence, with the International Diabetes Federation estimates of more than 100 million people with diabetes in India by 2030, 90\% of which will be type 2 diabetes [6]. While the prevalence of diabetes is slightly higher than in the rest of the world (9.1\% vs. $8.3 \%$ worldwide), studies have indicated that there are large regional and socioeconomic differences in diabetes prevalence in India.

More than 300 risk variants associated with type 2 diabetes and more than 50 with type 1 diabetes have been identified thus far, mostly based on studies in populations of European origin [1]. Many of these have been replicated in the Indian population including those in TCF7L2, MC4R, PPARG, KCNJ11, SLC30A8, HHEX, CDKN2A, IGF2BP2, CDKAL1 and MTNR1B [7]. Notably, certain genotypes (such as the Pro12Ala polymorphism of the PPAR $\gamma$ gene) that protect against the development of T2DM in the Caucasian population, does not seem to do so among Asian Indian patients [8]. A few loci associated with T2D in Indian studies have not been replicated in European populations thus far, including variants at TMEM163, SLAMF1 and ITLN1 loci [9,10]. Multiple previously reported T2D risk loci show a significant disparity in allele frequency across populations worldwide. These loci demonstrate extreme directional differentiation of risk allele frequencies across populations compared to the null distributions of European-frequency matched control alleles for other diseases. Most T2D risk alleles share a consistent pattern of decreasing frequencies along human migration into East Asia including Asian Indians [11]. Considering these differences, it is entirely plausible that differences in etiology might also exist between European and Indian populations [12].

Diabetes is still diagnosed based on one metabolite; glucose measured at fasting and $2 \mathrm{~h}$ post glucose/food intake. Moreover, Asian Indians are at a higher risk of developing T2D at a younger age and at lower BMI compared to patients from western countries. They are also at a higher risk for coronary artery disease and lower risk for microvascular complications compared to the western population. These findings hint towards a specific "Asian Indian phenotype" or the "thin-fat phenotype", which is a conglomerate of clinical and biochemical features that predispose Asian Indians to higher T2D risk [13]. A prime example of this is that for a given BMI, Asian Indians have higher waist circumference, higher waist-hip ratio, and higher visceral and subcutaneous fat leading to more insulin resistance compared to individuals of European origin $[14,15]$.
The cost of diabetes worldwide was US\$1.31 trillion, or $1.8 \%$ of the global gross domestic product (GDP), in 2015, two-thirds of which were direct medical costs, and one-third being indirect costs due to loss of productivity [16]. In India, the economics of healthcare is almost exclusively managed by the private sector, considering that the public health care funding is lower in India than other countries in the region, and considerably lower than in Europe and western countries. This means that the large economic burden of diabetes is almost exclusively borne by the patients [17].

This substantiates a need for better dissection of diabetes subtypes to facilitate better treatment strategies tailored for individual needs. Potential replication of the ANDIS clusters could help to understand etiology and identify individuals at greater risk for complications. Identification of novel clusters will be even more interesting to understand the complex etiology of diabetes in this largely heterogeneous population. However, this does not preclude the continued implementation of current treatment strategies and regimen by expert physicians. The new classification will, in fact, mandate the physicians to tailor therapies more suited to the patient's needs.

\section{References}

[1] Prasad RB, Groop L. Genetics of type 2 diabetes-pitfalls and possibilities. Genes 2015;6(1):87-123.

[2] Brasacchio D, et al. Hyperglycemia induces a dynamic cooperativity of histone methylase and demethylase enzymes associated with gene-activating epigenetic marks that coexist on the lysine tail. Diabetes 2009;58(5):1229-36.

[3] Ahlqvist E, et al. Novel subgroups of adult-onset diabetes and their association with outcomes: a data-driven cluster analysis of six variables. Lancet Diabetes Endocrinol 2018;6(5):361-9.

[4] Moorjani P, et al. Genetic evidence for recent population mixture in India. Am J Hum Genet 2013;93(3):422-38.

[5] Metspalu M, et al. Shared and unique components of human population structure and genome-wide signals of positive selection in South Asia. Am Hum Genet 2011;89(6):731-44.

[6] Diabetes Atlas : www.idf.org/diabetesatlas 2018.

[7] Chauhan G, et al. Impact of common variants of PPARG, KCNJ11, TCF7L2 SLC30A8, HHEX, CDKN2A, IGF2BP2, and CDKAL1 on the risk of type 2 diabetes in 5,164 Indians. Diabetes 2010;59(8):2068-74.

[8] Radha V, et al. Role of genetic polymorphism peroxisome proliferatoractivated receptor-gamma2 Pro12Ala on ethnic susceptibility to diabetes in South-Asian and Caucasian subjects: evidence for heterogeneity. Diabetes Care 2006;29(5):1046-51.

[9] Tabassum R, et al. Common variants of SLAMF1 and ITLN1 on 1q21 are associated with type 2 diabetes in Indian population. J Hum Genet 2012;57(3): 184-90.

[10] Tabassum R, et al. Genome-wide association study for type 2 diabetes in Indians identifies a new susceptibility locus at 2q21. Diabetes 2013;62(3): 977-86.

[11] Chen R, et al. Type 2 diabetes risk alleles demonstrate extreme directional differentiation among human populations, compared to other diseases. PLoS 
Genet 2012;8(4). e1002621.

[12] Holliday EG. Hints of unique genetic effects for type 2 diabetes in India. Diabetes 2013;62(5):1369-70.

[13] Unnikrishnan R, Anjana RM, Mohan V. Diabetes mellitus and its complications in India. Nat Rev Endocrinol 2016;12(6):357-70.

[14] McKeigue PM, Shah B, Marmot MG. Relation of central obesity and insulin resistance with high diabetes prevalence and cardiovascular risk in South
Asians. Lancet 1991;337(8738):382-6.

[15] Yajnik CS, et al. Neonatal anthropometry: the thin-fat Indian baby. The Pune maternal nutrition study. Int J Obes Relat Metab Disord 2003;27(2):173-80.

[16] Bommer C, et al. The global economic burden of diabetes in adults aged 20-79 years: a cost-of-illness study. Lancet Diabetes Endocrinol 2017;5(6):423-30.

[17] Atun R, et al. Diabetes in sub-Saharan Africa: from clinical care to health policy. Lancet Diabetes Endocrinol 2017;5(8):622-67.

Please cite this article in press as: Prasad RB, et al., Heterogeneity of diabetes - An Indian perspective, Diabetes \& Metabolic Syndrome: Clinical Research \& Reviews (2018), https://doi.org/10.1016/j.dsx.2018.07.001 